\title{
Assess the Impact of Obesity on Pregnancy and Neonatal Outcomes among Saudi Women at King Abdulaziz Medical City Jeddah
}

Running Title: Obesity impact on Saudi Pregnancy Outcomes

\author{
Authors \\ Nadia Adwani ${ }^{1}$, Howieda Fouly ${ }^{2 *}$, Tagwa Omer ${ }^{3}$ \\ ${ }^{1}$ Master degree of Midwifery, RN. Midwife at Adm Hospital - Ministry of Health, Jeddah, KSA \\ ${ }^{2} \mathrm{PhD}$. RN, Assistant professor- Maternity Nursing, College of Nursing, King Saud Bin Abdul-Aziz \\ University for Health Sciences, Jeddah KSA- Associate prof. Faculty of Nursing Assiut University Egypt. \\ ${ }^{3} \mathrm{PhD}$. RN. Associate professor at Nursing College of Nursing, King Saud Bin Abdul-Aziz University for Health \\ Sciences- Jeddah, KSA
}

\begin{abstract}
Background: The rising prevalence of obesity has a significant impact on obstetrics practice regarding maternal and perinatal complications includes recurrent miscarriage, pregnancy-induced hypertension, preeclampsia, gestational diabetes, and prolonged labor. Objective: To assess the impact of obesity on pregnancy and neonatal outcomes among Saudi women. Methods: The study was conducted at King Abdul-Aziz Medical City, Jeddah. Design: A quantitative research, crosssectional retrospective design. A total number of 186 participants were recruited from July to Dec.2018 according to eligibility criteria. The data were collected retrospectively by a review of the chart records of the labor and delivery department. Results: The mean (SD) age of participants was 31.94(5.67) years old; two-thirds were in obesity class1. There was a significant association between obesity and pre-existing thyroid disease and induced hypertension class 3 . However, Episiotomy showed that obesity class 3 was significantly different from obesity class 2. Conclusion: This study concludes obesity affects the outcomes of pregnant Saudi associations between obesity and preeclampsia, perineal tears, and episiotomy variables, and other variables reflect no associations. Recommendations: Further studies are needed to generalize the results.
\end{abstract}


This study endorses the pregnant women start the antenatal follow-up from 1st trimester so, the data will be available on the system for research.

Keywords: Obesity, BMI, maternal outcomes, neonatal outcomes.

What is known about the subject

- Obesity effect on pregnancy outcomes

- Obesity is accompanied by many diseases with pregnancy, such as DM, heart disease, and hypertension.

- Obesity effect on the fetal and neonates 


\section{What are the new findings of this study}

- The impact of obesity on pregnant Saudi women is associated with specific co-factors such as preeclampsia, perineal tears, and Episiotomy.

- There is no association between obesity and maternal gestational diabetes, preterm labor, induction or augmentation of labor, prolonged labor, emergency cesarean delivery, and postpartum hemorrhage.

- There is no association between obesity and neonatal IUGR, IUFD, preterm baby, stillbirth, neonatal mortality, and NICU admission.

\section{Introduction}

In recent years, obesity has begun to be considered a global health problem. It is the fifth leading cause of death worldwide. Obesity is a condition of abnormal and excessive fat accumulation in adipose tissue, leading to adverse health effects. The significant contributors to weight gain, which may eventually lead to obesity, are decreased physical activity, increased dietary fat intake, and genetic factors (DeNicola et al. 2015). The rise in obesity is associated with advanced age, which becomes apparent when considering the decrease in older adults' physical activity and metabolic processes. Marital status, high educational level, alcohol use, and high socioeconomic status are other factors associated with obesity (Paul et al. 2010).

Overweight and obesity have become the most general nutritional problems globally, as they impose significant burdens on health care systems. Obesity affects 2.1 billion people (almost one third) in the world. If the current trend continues, this figure may reach nearly half of the world's adult population by 2030 (Saudi guidelines 2016). 
Obesity is measured using various methods, including body mass index (BMI), waist circumference (WC), waist-hip ratio, skinfold, and percent body fat measurements. The majority of studies use BMI to measure obesity, while a few studies use WC (Musaiger et al., 2011). BMI is the most frequently used diagnostic tool in the current classification system of obesity (GómezAmbrosi et al. 2012). It is calculated by dividing weight in kilograms by height in square meters. The World Health Organization (WHO) divided BMI values into six categories to define different body weights, from underweight to obesity. These categories are: underweight (less than 18.5), average weight (18.5-24.9), overweight (25.0-29.9), obesity class I (30.0-34.9), obesity class II (35.0-39.9), and obesity class III (40.0 or greater) (Othman et al. 2018).

In addition to BMI and west circumference, it can be used to assess the abdominal fat and risk of obesity-related comorbidities, particularly cardiovascular disease and diabetes $\mathrm{WC}$ is determined using a measuring tape after asking the person to stand with their feet close together (about 12-15 $\mathrm{cm})$, distribute their weight equally, and breathe normally. The measuring tape should be held horizontally at a level midway between the lower rib margin and the iliac crest, approximately in line with the umbilicus. It should be loose enough to allow the measurer to place one finger between the measuring tape and the person's body. Finally, the measurement is recorded on an exhalation (Saudi Ministry of Health, 2014 ).

Obesity is associated with multiple diseases and may result in the death of millions of people every year. The risk of noncommunicable diseases (NCD), such as hypertension (HTN), prediabetes, type 2, diabetes mellitus (T2DM), dyslipidemia, and cardiovascular disease (CVD) increases significantly with obesity. In addition, obstructive sleep apnea and osteoarthritis relate to obesity. ${ }^{[8]}$ 
Females were to have a higher rate of obesity. Its prevalence doubled between 1980 and 2008, from $8 \%$ in 1980 to $14 \%$ in 2008 . The highest incidence of overweight and obesity in 2013 was in North Africa and the Middle East, where more than $65 \%$ of reproductive age females were overweight or obese (Aljabri et al 2018).

Maternal obesity is one of the central risk factors for adverse pregnancy outcomes, including gestational diabetes mellitus (GDM), operative delivery, and stillbirth. ${ }^{[9]}$ In brief, obesity is a known risk factor for many health problems. Obese women have a higher risk of complications during pregnancy and delivery, and obesity may hurt their offspring's health. According to a study of overweight and obesity in Saudi women of childbearing age, the following rates of obesity were found: $22.4 \%$ were obesity class I, $11.1 \%$ were obesity class II, and $6.6 \%$ were morbidly obese (obesity class III) (Aljabri, et al 2018). Even with the availability of updated data about the prevalence of obesity in the Kingdom of Saudi Arabia, there is a lack of research conducted in Saudi Arabia about the outcome of obese pregnant women.

\section{Methodology}

This study was conducted in the labor room at King Khaled Hospital in Jeddah. The hospital affiliated to the Ministry of National Guard Health Affairs in the Western Region is a 600 bedded, Joint Commission International (JCI) accredited Tertiary Level Hospital. Currently, ten admission beds within the labor and delivery ( 6 beds for active labor, three beds for induction of labor, and one bed for operation room). The total number of deliveries per year was 3161 .

This study included all obese pregnant women primigravida and multigravida, age 18 to 44 years old, and the BMI for those pregnant women was 30 or more. All pregnant attended the antenatal 
clinic during the first trimester and delivered through expected vaginal delivery at King Khaled Hospital. Our study excluded all multiple pregnancies and medical conditions. For example, all pregnant who had diabetes mellitus before pregnancy, had chronic hypertension before pregnancy, had a history of cardiac diseases or the current pregnancy with abnormal fetus lie like a breach or transverse lie, and all elective cesarean section excluded. This study was quantitative research, cross-sectional cohort study retrospective design, which involves collecting data at one point in time. The phenomena of the course were captured during one data collection period. Crosssectional arrangements are especially appropriate for describing the status of phenomena or relationships among phenomena at a fixed point (Davies-tuck, et al. 2016). In retrospective design, the phenomenon observed in the present is linked to phenomena occurring in the past. This study described the outcomes of obese pregnancy during the antenatal, intrapartum period, and the neonatal outcome at the delivery time.

Data was collected through a nonprobability sampling design retrospectively by reviewing the chart records of the labor and delivery department. The study included all obese women who delivered in the selected six months. Then compared the outcomes of pregnancy through the different obesity' classes. The data was collected from the chart using the BEST Care 2.0 system, founded in all the hospital computers. The total number of deliveries in the selected six months, during the period from $01 \backslash 07 / 2018$ to $31 \backslash 12 \backslash 2018$, was 1748 delivery. After excluding the patients of pregnant women who did not meet the criteria, the total number of patients included in this study were 186 patients, which is the total sample

The data analyzed using the Statistical Package of Social Sciences (SPSS) version 23. Proper statistical tests were used to describe the finding of the study and to achieve the objectives of the 
study, with appropriate statistical measures and tests. The ordinal and nominal variables were presented in the form of frequencies and percentages. Chi-square, which is a statistical test, was used to examine the association between two quantitative variables. P-value of less than 0.05 was considered statistically significant, and it can be concluded that there is a relationship between the two variables. Fisher's exact test was performed to test the significance of the difference in proportions; it is used for small sample size or when the cells in the contingency table have no observations. Post hoc test was also used for comparing all possible pairs of groups following a significant test of overall group differences (e.g., chi-square) ( Polit and Beck 2010). 


\section{Results}

Table 1 presents respondents' basic characteristics; the majority are $28.5 \%$ of women aged between 25 to 29 years, and only $12.4 \%$ for the age group between 40 to 45 years. Regarding the obstetric characteristics, results revealed that primigravida was only $16.7 \%$, while the majority were multigravida' $83.3 \%$. The mode of previous delivery $67.7 \%$ were delivered through SVD and $14.0 \%$ delivered by CS. Two-thirds of women were in class 1 obesity (BMI 30-34), while $7.5 \%$ of them in obesity class 3 (BMI more than 40). Regarding the risk factors, only $2.2 \%$ were smokers, $8.6 \%$ have pre-existing thyroid disease and $5.4 \%$ had recurrent miscarriages.

Table 2 shows the frequency and result of fisher's exact test that examined the relationship between obesity classes and co-factors. The pre-existing thyroid disease shows that $16(12.5 \%)$ cases found in obesity class1. There was a significant association between the obesity and pre-existing thyroid disease at $95 \%$ level of confidence, and $P=0.015$. For preeclampsia, one case only $(7.1 \%)$ out of $14(92.9 \%)$ found in class 3 obesity. There was a significant association between obesity and preeclampsia at $90 \%$ level of confidence, the $P=0.075$. For perineal tears, intact perineal at delivery was $55(43.0 \%)$ cases, while $45(35.2 \%) 24(18.8 \%)$ had first and second-degree tears respectively. For obesity class 2, 24 women (54.5\%) deliver with intact perineum, 13 (29.5\%) and $4(9.1 \%)$ cases had $1^{\text {st }}$ and second-degree tears respectively. In obesity class3 the highest percentage $6(42.0 \%)$ deliver with intact perineal, while tears were $4(28.6 \%)$, and $(7.1 \%)$ cases had first and second-degree tears respectively. There is a significant association between the obesity and perineal tears at $95 \%$ level of confidence, and $P=0.020$. For Episiotomy. In obesity class2, $3(6.8 \%)$ cases out of $41(93.2 \%)$ had Episiotomy and in obesity class3, $3(21.4 \%)$ out of 
11(78.6\%) had Episiotomy. There is a significant association between the obesity and Episiotomy at a $95 \%$ level of confidence. The P-value of the test is 0.037 .

Table 3. Shows the result of post-hoc tests that were conducted to test pairwise comparisons. The finding indicates that Pre-existing thyroid disease compared to obesity class1 was significantly different from obesity class $2(p=0.033)$. Obesity class 1 and obesity class 3 , and both obesity class 2 and class 3 were not significantly different.

The comparison of induced hypertension shows that obesity class 3 was significantly different from obesity class $1(p=0.001)$. Furthermore, obesity class3 was significantly different from obesity class2 $(p=0.001)$. On the other hand, obesity class1 and class2 were not significant in regard to induced hypertension. The finding of perineal tears shows that obesity class1 was significantly different from obesity class $3(p=0.020)$. Either obesity class 1 and obesity class 2 or obesity class 2 and class 3 were not significantly different. The result of Episiotomy shows that obesity class 3 was significantly different from obesity class $2(p=0.044)$. Also, obesity class 3 was significantly different from obesity class $1(\mathrm{p}=0.008)$. Besides, there is no a statistically significant difference between obesity class 2 and class 1.

\section{Discussion}

Obesity has become a globally common problem due to change in lifestyle. The accumulation of excess body fat can cause a serious issue in both health and longevity, because of the high risk to have hypertension, diabetes, stroke, heart disease, and cancer among obese individuals. Despite including the obesity in the International Classification of Diseases in 1948, it was not recognized as a global epidemic by the WHO until 1997. Universally, there were more than 600 million obese adults in 2014 (Zutshi, et al 2018). 
The rising prevalence of obesity has a significant impact on obstetrics practice. Maternal complication association due to obesity includes recurrent miscarriage, pregnancy-induced hypertension, preeclampsia, gestational diabetes, prolonged labor, and increased risk of interventions like induction of labor, operative delivery, shoulder dystocia, and postpartum hemorrhage (Sinha et al. 2018). On the other hand, perinatal complications include birth defect like congenital anomalies, macrosomia, stillbirth, preterm birth, and need for admission to the neonatal intensive care unit (Sinha et al 2018).

Our study aimed to assess the impact of obesity on pregnancy and neonatal outcomes among Saudi women. This study's first objective was to describe the pregnancy and the neonatal outcomes for obese pregnant women. The present study revealed a significant association between obesity and pre-existing thyroid disease, pregnancy-induced hypertension (preeclampsia), perineal tears, and Episiotomy.

Regarding the association between obesity and pre-existing thyroid disease, our result shows a statistically significant association between obesity and pre-existing thyroid disease, similarly, in a prospective cohort study done by (Collares et al. 2017) conducted at Rotterdam, the Netherlands. The study aimed to examine maternal thyroid function associations in early pregnancy with maternal BMI and weight gain during pregnancy. This study identified a higher maternal TSH level in early pregnancy associated with a higher prepregnancy BMI and an increased risk of excessive gestational weight gain. In contrast, a higher maternal FT4 level was associated with a lower prepregnancy BMI and a lower risk of excessive gestational weight gain. Associations of maternal thyroid function with gestational weight gain were strongest for weight gain in early pregnancy. Also, (Pop et al. 2013), in a prospective follow-up study of thyroid parameters and 
gestational weight gain, indicated that higher median TSH and lower median FT4 levels in all trimesters were correlated with a higher amount of total weight gain during pregnancy.

Conversely, a study conducted at Baylor aimed to measure T3, fT4, and TSH in maternal and matched cord blood serum from average weight, overweight and obese gravidae to determine alterations in maternal and neonatal TH levels by maternal obesity. The result showed no significant difference in gestational age and weight gain (Kahr et al. 2016).

Regarding the association between obesity and preeclampsia, our result shows a statistically significant association between obesity and preeclampsia, similarly, in prospective cohort research conducted in Jeddah Maternity and Children Hospital $(\mathrm{MCH})$. The study revealed a positive association between obesity and increased risk of pregnancy-induced hypertension and preeclampsia compared with the normal-weight women. A previous study by Zayed et al. (2018) conducted in Tabuk City found a higher risk of preeclampsia in obese non-GDM women than in non-obese GDM women.

Likewise, (Young et al. 2016) demonstrated in the retrospective cohort study that women with prepregnancy obesity are more likely to develop preeclampsia, which reported a statistically significant association between obesity and preeclampsia.

According to Marchi et al. 2015), in a review that aimed to summarize the findings of published systematic reviews regarding the possible risks for pregnant women with obesity and their infants. The review demonstrates an association between obesity and gestational hypertension and preeclampsia, identified as a risk factor in 54 studies.

A cohort study was done by Paré (2014), where data was collected from three large urban academic centers, the result revealed a positive association between obesity and preeclampsia. 
Moreover, a study by (Salihu et al. 2012) reported in the systematic literature review of two decades (1992-2011) that obesity has an association with preeclampsia or hypertension during pregnancy.

On the other hand, the findings reported by Gaillard et al. 2016 in a prospective cohort study, the result shows that higher gestational weight gain was associated with a higher risk of pregnancyinduced hypertension, but not with preeclampsia. Additionally, higher prepregnancy BMI was associated with high blood pressure both systolic and diastolic in all trimesters.

Regarding perineal tears, our result shows a statistically significant association between obesity and perineal tears. the association between these two variables is a significant negative association, which means when the obesity class decrease the risk for perineal tears increased. Conversely, when the obesity class increased the risk for perineal tears decreased.

Similarly, Garretto et al. 2016 in the case control study revealed no significant association between obesity and perineal tears. Likewise, a previous cohort study by Blomberg, 2014 which demonstrated that the risk of partial anal sphincter injury or total sphincter injury, and fourthdegree perineal tears decreased with maternal obesity. The general risk for any anal sphincter injury among obese women class 3 was reduced with $25 \%$ compared to normal women weight.

Previous studies found that obese women have lower risk for perineal tears which known as a protective effect of obesity. The negative association between obesity and perineal tears has shown that obesity in pregnancy is not commonly associated with adverse events (Durnea, et al. 2017).

The findings of Al-ghamdi, et al 2018 in a retrospective cohort study conducted in Riyadh, Saudi Arabia indicated that there was an association between obesity and perineal tears. 
Furthermore, the prospective cohort study by (Kamel, et al. 2018), which carried out in Egypt, reported that obese women had a higher rate of perineal tear, mostly second-degree tearing, than those with normal BMI. Besides, there was no significant difference in the incidence of the thirddegree perineal tear in obese women.

In the same line, Lindholm and Altman (2013) found that the rate of third- and fourth-degree perineal tears decreased with increasing BMI, whereas the opposite was true for first- and seconddegree perineal tears, which increased with increasing BMI.

In conclusion, the incongruence between our study and those studies may be due to the large study population, and the data included an ethnically heterogeneous population.

Regarding the Episiotomy, our result shows a statistically significant association between obesity and Episiotomy. Dissimilarly, the case-control study (BraGa et al. 2014) conducted on pregnant women who submitted to Episiotomy in Brazil reported no association between obesity and Episiotomy.

In the same line, a study done by Anzaku, Idikwu, \& Emmanuel (2015) reported in a prospective cohort study conducted at Bingham. that there was no increased risk of episiotomy/perineal tear among obese pregnant women.

Similarly, a systematic review and meta-analysis were conducted to investigate maternal obesity in Africa by (Onubi et al. 2015) and reflected that there was no significant relationship between maternal obesity and Episiotomy or perineal tear. The contrast between our study and those studies may be due to the ethnically heterogeneous population. 
This study's second objective was to compare the pregnancy and the neonatal outcomes of obese pregnant women in different obesity classes. Regarding preeclampsia, our study's findings were in accordance with other findings reported by (Young, et al 2016), which stated that preterm preeclampsia is high in women with class III obesity compared to normal-weight women. This agrees with only one case in our study, which was categorized under obesity class3.

Several previous studies [Othman et al 2018; Zayed et al. 2017; Marchi et al. 2015; Salihu et al. 2012; Blomberg et al. 2014; Onubi et al. 2016] reflected their result based on a comparison of obesity in general with average weight not like our study the comparison in obesity classes.

\section{Conclusion \& Implication for future practice}

Obesity is a severe public health problem and harms maternal pregnancy outcomes. This study reflected the impact of obesity on pregnant Saudi women through several associations between obesity and specific co-factors as preeclampsia, perineal tears, and episiotomy variables. However, the study concludes other variables reflect no associations. Hence, there is no association between obesity and maternal outcomes like gestational diabetes, preterm labor, induction or augmentation of labor, prolonged labor, emergency cesarean delivery, and postpartum hemorrhage. Also, there is no association between obesity and neonatal outcomes like IUGR, IUFD, preterm baby, stillbirth, neonatal mortality, and NICU admission.

Recommendations: Further studies are needed to conduct a larger sample size and include the elective cesarean section and its relation to obesity. This study encourages the broadness of data to have all Jeddah districts, not only one point and increases the data collection period to generalize the results. The study also recommends the pregnant women start the antenatal follow-up from 1 st trimester so the data will be available on the research system. 


\section{References}

Al-ghamdi, T., Chamsi, A. T., \& Mardawi, E. Al. (2018). Incidence and Risk Factors for Development of Third and Fourth Degree Perineal Tears : A Four Year Experience in a Single Saudi Center Journal of Women' s Health Care, 7(2), 2-5. https://doi.org/10.4172/2167$\underline{0420.1000423}$

Aljabri, K. S., Bokhari, S. A., Khan, Alshareef, Ebu Elsaoud, H. M., Aljabri, B. K., Boraie, R. E. . (2018). Ec Endocrinology and Metabolic Research Research Article Overweight and Obesity in Saudi Women of Childbearing Age, 2, 53-62.

Anita Zutshi, Jayasree Santhosh, Julie Sheikh, Fareeha Naeem, Ahmed Al-Hamedi, Shahla Khan, E. A.-S. (2018). Implications of Early Pregnancy Obesity on Maternal, Fetal and Neonatal Health, 18(February), 47-53. https://doi.org/10.18295/squmj.2018.18.01.008

Anzaku, A. S., Idikwu, O. G., \& Emmanuel, O. A. (2015). Impacts of Obesity on Maternal and Fetal Outcomes in Women with Singleton Pregnancy at a Nigerian Clinical Setting Impacts of Obesity on Maternal and Fetal Outcomes in Women with Singleton Pregnancy at a Nigerian Clinical Setting, (January 2016). https://doi.org/10.9734/BJMMR/2015/15618

Blomberg, M. (2014). Maternal Body Mass Index and Risk of Obstetric Anal Sphincter Injury, 2014.

BraGa, G. C., ClemenTino, suelem T. pereira, Luz, paTríCia F. neves da, SCavuzzi, A., NeTo, C. noronha, \& Amorim, melania maria ramos. (2014). Risk factors for episiotomy : a casecontrol study, 60(5), 465-472.

Collares, F. M., Korevaar, T. I. M., Hofman, A., Steegers, E. A. P., Peeters, R. P., \& Jaddoe, V. 
W. V. (2017). Maternal thyroid function, prepregnancy obesity and gestational weight gain - The Generation R Study: A prospective cohort study, (May), 799-806. https://doi.org/10.1111/cen.13412

Constantin M. Durnea, Ali E. Jaffery, Nivedita Gauthaman, S. K. D. (2014). Effect of body mass index on the incidence of perineal trauma. https://doi.org/10.1111/ijlh.12426

Davies-tuck, M., Mockler, J. C., Stewart, L., Knight, M., \& Wallace, E. M. (2016). Obesity and pregnancy outcomes : Do the relationships differ by maternal region of birth ? A retrospective cohort study. BMC Pregnancy and Childbirth, 1-8. https://doi.org/10.1186/s12884-016$\underline{1087-5}$

DeNicola, E., Aburizaiza, O. S., Siddique, A., Khwaja, H., \& Carpenter, D. O. (2015). Obesity and public health in the Kingdom of Saudi Arabia. Reviews on Environmental Health, 30(3), 191-205. https://doi.org/10.1515/reveh-2015-0008

E. S. Lindholm and D. Altman. (2013). "Risk of obstetric anal sphincter lacerations among obese women," BJOG: An International Journal of Obstetrics \& Gynaecology, vol. 120, no. 9, pp. $1110-1115,2013$.

Gaillard, R., Steegers, E. A. P., Hofman, A., \& Jaddoe, V. W. V. (2011). Associations of maternal obesity with blood pressure and the risks of gestational hypertensive disorders . The Generation R Study, 937-944. https://doi.org/10.1097/HJH.0b013e328345500c

Garretto, D., Lin, B. B., Syn, H. L., Judge, N., Beckerman, K., Atallah, F., Bernstein, P. S. (2016). Obesity May Be Protective against Severe Perineal Lacerations, 2016.

Gómez-Ambrosi, J., Silva, C., Galofré, J. C., Escalada, J., Santos, S., Millán, D., ... Frühbeck, G. (2012). Body mass index classification misses subjects with increased cardiometabolic risk factors related to elevated adiposity. International Journal of Obesity, 36(2), 286-294. 


\section{https://doi.org/10.1038/ijo.2011.100}

Kahr, M. K., Antony, K. M., Delbeccaro, M., Hu, M., Aagaard, K. M., \& Suter, M. A. (2016). Increasing maternal obesity is associated with alterations in both maternal and neonatal thyroid hormone levels, 551-557. https://doi.org/10.1111/cen.12974

Kamel, H. A. H., Ibrahim, A. S. M., \& Abdo, M. M. (2018). Maternal Obesity and Its Effect in Late Pregnancy and Labour, 71(April), 2982-2988.

Kavita Sinha, , Subhash Pandey, C. R. Das. (2016). Impact of Maternal Obesity on Pregnancy Outcome, (October 2018). https://doi.org/10.3126/jngmc.v14i2.21531

Marchi, J., Berg, M., Dencker, A., Olander, E. K., \& Begley, C. (2015). Risks associated with obesity in pregnancy, for the mother and baby: A systematic review of reviews. Obesity

Musaiger, A. O. (2011). Overweight and obesity in Eastern Mediterranean Region: Prevalence and possible causes. Journal of Obesity, 2011(May). https://doi.org/10.1155/2011/407237

Othman, M., Himayda, S. S., \& Shaaban, L. (2018). Obesity and Pregnancy in Saudi Women. Arch Clin Med Case Rep, 2(2), 50-55. https://doi.org/10.26502/acmcr.96550024

Onubi, O. J., Marais, D., Aucott, L., Okonofua, F., \& Poobalan, A. S. (2015). Maternal obesity in Africa : a systematic review and meta-analysis, 38(3). https://doi.org/10.1093/pubmed/fdv138

Paul, E., Mtumwa, A. H., Ntwenya, J. E., \& Vuai, S. A. H. (2016). Disparities in Risk Factors Associated with Obesity between Zanzibar and Tanzania Mainland among Women of Reproductive Age Based on the 2010 TDHS, 2016.

Paré, E., Parry, S., Newton, A., Lim, K., Mcelrath, T. F., \& Pucci, D. (2014). Clinical Risk Factors for Preeclampsia in the 21st Century, 124(4), 763-770. https://doi.org/10.1097/AOG.0000000000000451 
Polit, D. ., \& Beck, C. T. (2010). Essentials of Nursing Research Evidence for Nursing Practice.

Pop VJ, Biondi B, Wijnen HA, Kuppens SM, Lvader H (2013). Maternal thyroid parameters, body mass index, and subsequent weight gain during pregnancy in healthy euthyroid women. Clin Endocrinol (Oxf). 2013;79:577-583.

SALIHU, H. M., CRUZ, C. D. LA, RAHMAN, S., \& AUGUST, E. M. (2012). Does maternal obesity cause preeclampsia? A systematic review of the evidence, (May 2014).

Saudi guidelines on prevention and management of obesity./ Ministry of health - Riyadh ,(2016). 51p;..cm ISBN : 978-603-8174-89-0

Saudi Ministry of Health. The National Guidelines for Management of Cardio-metabolic Risk Factors in Primary Health care, 2014

Young, O. M., Twedt, R., \& Catov, J. M. (2016). Prepregnancy Maternal Obesity and the Risk of Preterm Preeclampsia in the American Primigravida, 24(6), 1226-1229. https://doi.org/10.1002/oby.21412.

Zayed, E. S., Allah, R. K. F., Shaman, A. A., Yousef, R. S., Elsaifi, O., \& Bakheit, K. H. (2018). The Effects of Maternal Obesity and Gestational Diabetes on the Pregnancy Outcomes (HAPO) In Saudi Women, At Tabuk City, https://doi.org/10.7537/marslsj141017.04 